\title{
A dynamic analysis of viral load,T lymphocyte subsets and main biochemical indexes before and after prevention of mother to child transmission in HIV/AIDS
}

\author{
Guosheng $\mathrm{Su}^{1,2}$, Yesheng $\mathrm{Wei}^{3^{*}}$, Lihua Qin ${ }^{4 *}$, Lida Mo ${ }^{5}$ \\ ${ }^{I}$ Department of Clinical Laboratory, the First Affiliated Hospital of Jinan University, Guangzhou, Guangdong, China; \\ ${ }^{2}$ Department of Orthopedics, the Hospital of Traditional Chinese and Western Medicine of Guangxi Guigang City, Guigang Red Cross \\ Hospital, Guigang 537100, Guangxi, China; \\ ${ }^{3}$ Department of Clinical Laboratory, the First Affiliated Hspital of Guilin Medical College, Guilin 541000, Guangxi, China; \\ ${ }^{4}$ Department of Osteoarthritis Nursing and Department of Orthopedics, the Hospital of Traditional Chinese and Western Medicine of \\ Guangxi Guigang City, Guigang Red Cross Hospital, Guigang 537100, Guangxi, China; \\ ${ }^{5}$ Department of Clinical Laboratory, Nanning Fourth People's Hospital, Nanning Infectious Diseases Hospital Affiliated to Guangxi \\ Medical University, Guangxi AIDS Clinical Treatment Center, Nanning 530023, Guangxi, China.
}

\begin{abstract}
This study aims to investigate changes in viral load, T lymphocyte subsets and other main biochemical indexes of HIV/AIDS in the prevention of mother to child transmission (PMTCT). In this study, 152 pregnant women with HIV/AIDS enrolled into our hospital from January 2013 to June 2015 were chosen as objects. Changes in viral load, T lymphocyte subsets and other main biochemical indexes of HIV/AIDS were tested and compared before and after 3 months of PMTCT and in neonatals one week after birth. The CD4/CD8 examination result, and difference in CD4 before and after prevention(in the newborns after a week) was statistically significant $(P<0.05)$, and the rest showed no statistical significance. For the dynamic analysis of main biochemical test results: $\mathrm{K}^{+}, \mathrm{Na}^{+}$, $\mathrm{Cl}^{-}$, BG, OS, BUN, BUN/Cr, UA,TDIL, DBIL, TP, ALB, CK, LDH, HDL, LDL and other indexes before and after prevention attained statistical significances $(P<0.05$ or above).The same sample in the three groups was detected by repeated analysis of variance, $\mathrm{K}^{+}, \mathrm{Na}^{+}, \mathrm{Cl}^{-}$, BG, OS, BUN/Cr, UA, DBIL, ALB, CK, LDH, HDL, LDL and other indexes also showing $P$ at less than 0.05 or above, among which $\mathrm{K}^{+}, \mathrm{Cl}^{-}, \mathrm{CK}$, LDL showed homogeneity of variance, while $\mathrm{Na}^{+}, \mathrm{BG}$, OS, BUN/Cr, UA, DBIL, ALB, LDH, HDL showed unequal homogeneity of variance. The study suggest that the dynamic analysis of viral load, T lymphocyte subsets and main biochemical indexes before and after PMTCT in HIV/AIDS are important means to evaluate the dose and treatment of antiretroviral drugs. Monitoring of above indexes is helpful to judge and analyze the condition of the maternal body at various stages, so antiviral drug treatment can be adjusted.
\end{abstract}

Keywords: HIV/AIDS, prevention of mother to child transmission (PMTCT), viral load, T lymphocyte subsets count, main biochemical indexes.

*Correspondence to: Yesheng Wei, Department of Clinical Laboratory, the First Affiliated Hspital of Guilin Medical College, Guilin 530023, Guangxi, China. E-mail: 741106791@qq.com; Lihua Qin, Department of Osteoarthritis Nursing and Department of Orthopedics, the Hospital of Traditional Chinese and Western Medicine of Guangxi Guigang City,Guigang Red Cross Hospital, Guigang 537100, Guangxi, China. E-mail: 787209349@qq.com.

Conflict of interests: The authors declare that they have no competing interests. 


\section{INTRODUCTION}

One of the greatest challenges surrounding the HIV/AIDS virus is to prevent transmission from pregnant women to their unborn children ${ }^{[1-5]}$. Until now, efforts to prevent and control HIV / AIDS have resulted in a set of professional diagnosis and treatment methods: such as the joint use of Zidovudine (AZT) and DideoxyThio-Cytidine (3CT), Luo matching, Waley-Ritonavir (kritonShiba) and Nevirapine (NVP).

However, the side effects of antiviral drugs not only affect the normal development of the fetus, but may also cause fetal malformations ${ }^{[6-9]}$. For pregnant women, the side effects are mainly: poor blood circulation, liver and kidney function damage and gastrointestinal reaction $^{[10-11]}$. Therefore, this study aims to understand changes in the main biochemical indexes of viral load, and $\mathrm{T}$ lymphocyte subset count in women with the HIV/AIDS before and after prevention of mother to child transmission (PMTCT), so as to guide clinical medication. The changes of the viral load, T lymphocyte subsets count and other main biochemical indexes before and after maternal and infant prevention of HIV/ AIDS were analyzed dynamically. The results were reported as follows.

\section{MATERIALS AND METHODS}

\section{Objects of the study}

One hundred and fifty two cases of HIV/AIDS positive women undergoing pregnancy were selected from the hospital from January 2013 to June 2015. All selected cases were diagnosed for the first time and had not undergone any antiviral treatment. Their ages ranged from 17 to 39, at an average age of 28.01 and a standard deviation of 5.04. Their gestational time was between 1 to 6 months. The study had the consent of the medical ethics committee and informed consent from the patients themselves.

\section{Instruments and reagents}

The virus load was detected by the Roche COBAS Taqman48, while T lymphocyte subset counts were detected by BD FACSCalibur from BD biosciences (N.J. USA). The main biochemical indexes were detected by Roche Modular 6000?8000? PPE from Roche Diagnostics (I.N. USA). All the reagents were provided strictly by instruments company and used within the validity period of use. All the test items were carried out with strict quality control according to the requirements of the laboratory quality management manual. The samples were tested after reaching the set objectives' quality standards.

\section{Inclusion criteria}

The subjects in this study met following criteria: 1) Pregnancies with established perinatal health records, who were found infected with HIV during their first physical examination. 2) Having a continuously declining number of $\mathrm{CD} 4$ cells. 3) Confirmed without prior antiviral treatment by western Blot test. After the consent of the medical ethics committee and the agreement from patients themselves, they were included in the study.

\section{Diagnostic criteria}

The diagnostic criteria confirming the subjects with HIV/AIDS complied with the following. In addition to any one of the clinical manifestations, there was a history of epidemiology, and HIV antibodies were present in laboratory tests. If the HIV antibody tested positive and the HIV-RNA in plasma was positive, or one of them was positive with the $\mathrm{CD} 4^{+} \mathrm{T}$ lymphocyte count less than 200 cells / $\mu \mathrm{L}$, patients were also diagnosed as having AIDS. The fluorescence marker scatter diagram demonstrating CD3 and CD4 cells is shown in Fig.1 to Fig.3.

\section{Maternal and infant prevention method}

The method of maternal and infant prevention was
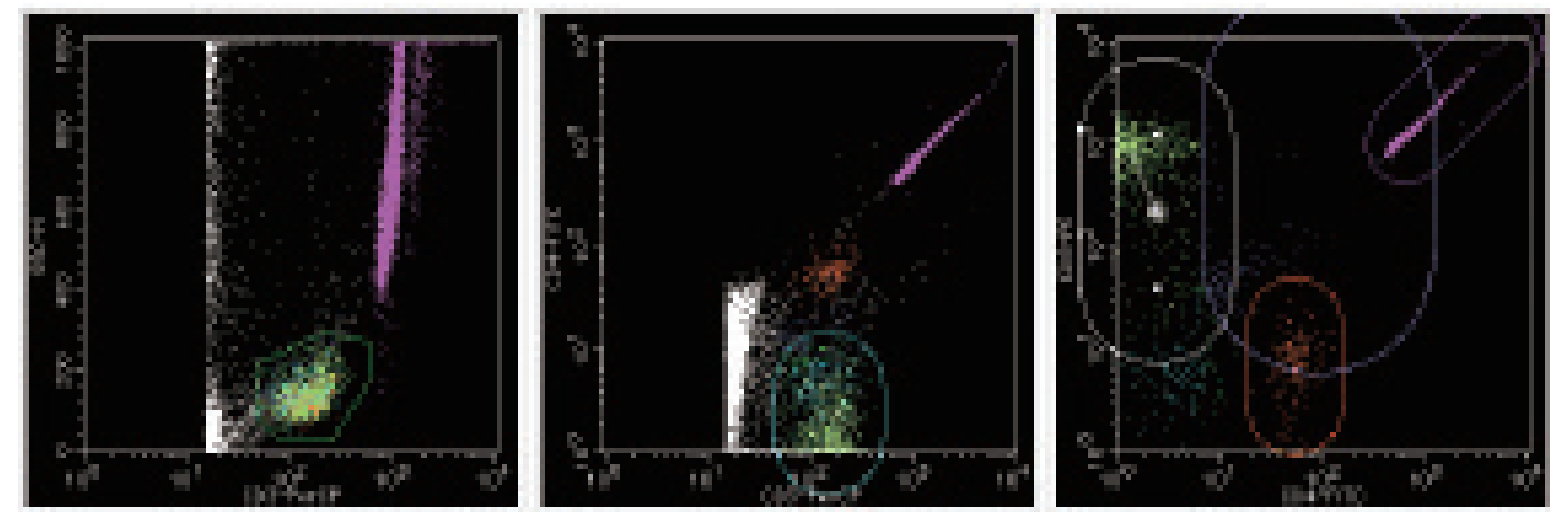

Fig1 Fluorescence marker scatter plots with reduced CD3 and CD4 results. 

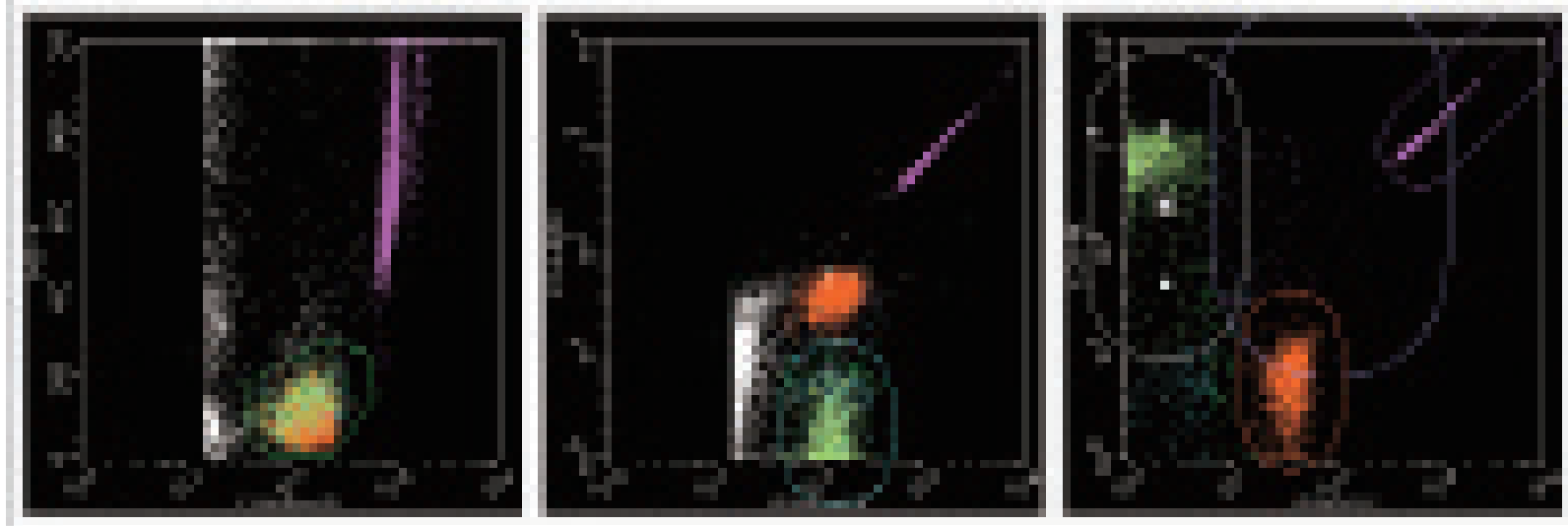

Fig.2 Fluorescence marker scatter plots with normal CD3 and CD4 results.
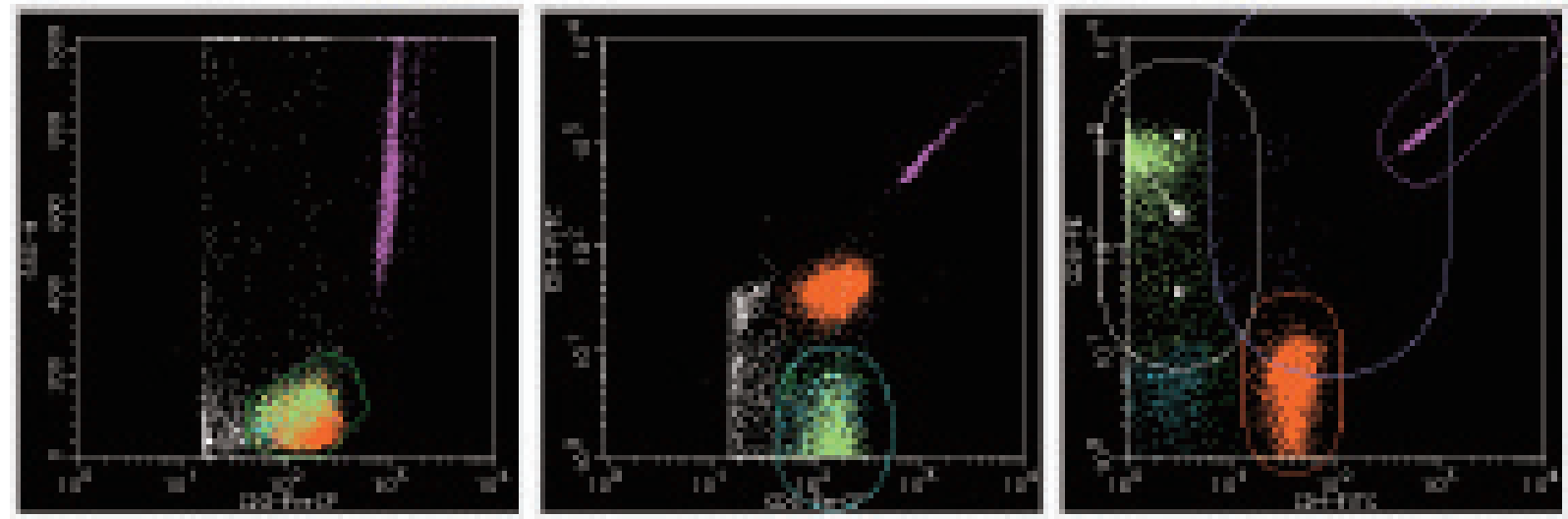

Fig.3 Fluorescence marker scatter plots with elevated CD3 and CD4 results.

carried out according to the standard as recommended by "The Chinese Guide for the Free Antiretroviral Treatment of AIDS" (Third Edition) 2010. Methods: AZT $0.3 \mathrm{~g} /$ time, 2 times daily. 3CT $0.3 \mathrm{~g} /$ time, once a day. KritonShiba tablets (each containing lopinavir $200 \mathrm{mg}$, ritonavir $50 \mathrm{mg}$ ) 2 tablets / time, twice a day. $0.2 \mathrm{mg} / \mathrm{kg}$ of NVP was added when close to giving birth, once orally. The delivery method was elective cesarean section. Changes in viral load, T lymphocyte subset count and other main biochemical indexes were observed. If the use of antiviral drugs was likely to cause liver or kidney function impairment, and / or an imbalance of electrolyte and acid-base, the relating clinical medication or treatment was regulated within the body's tolerance so as to not cause damage or compromise function.

\section{Detection methods of this subjects}

According to different detection regimens, a sufficient amount of venous blood was extracted from the pregnant mothers before and after 3 months of PMTCT and the newborn one week after birth. After extracting the blood, the virus load, T lymphocyte sub-population meter number and other main biochemical indexes were strictly detected according to the operation procedure.
The changes of the indicators were then observed, the results analyzed and relevant conclusions proposed.

\section{Statistical analysis}

Software SPSS 19.0 was used for statistical analysis. The direct counting method was applied for the analysis of HIV/AIDS pregnancy related cases in each group. In order to facilitate the statistical data, the viral load value less than $20 \mathrm{cp} / \mathrm{mL}$ was set as 20 . The measurement data in the groups before and after treatment is shown with $(\bar{x} \pm s)$. The comparison between the two groups before and after treatment was completed by paired test, while the $t$-test was used to compare the two random groups. Reproducible detection methods were used in duplicate in the three groups, and the data was analyzed with the repeatable test of variance. If $P$ was less than 0.05 , statistical significant difference was noted.

\section{RESULTS}

\section{Comparison of the detection results of viral load quantity of HIV/AIDS before and after PMTCT}

When comparing the viral load quantity in mothers before and after 3 months prevention and children one 
week after birth, $t=1.276$ and $P=0.101$ was recorded, which was not statistically significant. In the comparison between newborns after one week before and after prevention, $t=1.303$ and $P=0.097$ was recorded, which also not significant. For the comparison of results after 3 months prevention and neonatal birth of one week, again the difference was not found statistically significant $(t=1.110$ and $P=0.135)$. The same sample in three groups was detected by repeated analysis of variance $(P>0.05$, Table 1).

Comparison of the detection results of $\mathbf{T} \mathbf{l y m}-$ phocyte subsets of HIV/AIDS before and after PMTCT

The results of the comparison of $\mathrm{T}$ lymphocyte subsets counts of CD3 and CD8 before and after PMTCT, were not statistically significant, while the CD4 in newborns after one week before and after prevention,were found statistically significant $(P<0.05)$. In the comparison of CD4 and CD8 results from neonatal birth of one week after 3 months prevention, the difference was also statistically significant $(P<0.05)$. In a word, the above indicators before and after prevention, as well as the newborns of one week, the changes of CD4 and CD4/CD8 all had significant statistical difference $(P<0.05)$, while the others showed no significant statistical difference. The same sample in the three groups was detected by repeated analysis of variance $(P>0.05$, Table 1).

Table 1 Comparison of $\mathrm{T}$ Lymphocyte subsets count and viral load numbers test results before and after PMCTC in HIV

$(\bar{x} \pm s)$

\begin{tabular}{|c|c|c|c|c|c|c|}
\hline \multirow{2}{*}{ Group } & \multirow{2}{*}{ Cases } & \multicolumn{3}{|c|}{ 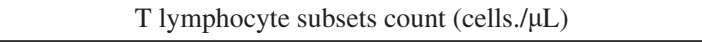 } & \multirow{2}{*}{$\begin{array}{c}\text { Ratio of CD4/ } \\
\text { CD8 }\end{array}$} & \multirow{2}{*}{$\begin{array}{c}\text { Viral load } \\
\text { (replica/ } \mu \mathrm{L})\end{array}$} \\
\hline & & CD3 & CD4 & CD8 & & \\
\hline before PMCTC & 152 & $1,162.84 \pm 522.44$ & $300.12 \pm 177.64$ & $839.04 \pm 441.26$ & $0.43 \pm 0.37$ & $1,7952.25 \pm 168,560.40$ \\
\hline 3 months after PMCTC & 152 & $1,202.37 \pm 484.67$ & $315.77 \pm 177.61$ & $824.77 \pm 413.88$ & $0.49 \pm 0.48$ & $499.85 \pm 3,737.89$ \\
\hline Newborns after one week & 152 & $1,203.70 \pm 469.25$ & $340.39 \pm 183.42$ & $798.49 \pm 397.97$ & $0.55 \pm 0.69$ & $1,40.51 \pm 1,450.19$ \\
\hline$F$ value of $\mathrm{CV}$ & - & 0.340 & 1.940 & 0.370 & 1.950 & 1.660 \\
\hline$P$ value & - & 0.714 & 0.145 & 0.692 & 0.144 & 0.191 \\
\hline
\end{tabular}

$\mathrm{CV}$ :comparison variance.

\section{Comparison of changes in main biochemical indexes before and after PMTCT of HIV/AIDS pregnant women}

Comparison of electrolyte and renal function in HIV/ AIDS pregnant mothers before and after PMTCT

For the comparison of the results of Kalium ion from newborns,before and after one week of PMTCT, there were significant statistical differences $(P<0.01)$, as was the comparison between prevention after 3 months $(P<0.01)$. The comparison of results after 3 months prevention and one week post birth was statistically significant $(P<0.01)$. Moreover, the comparison of Sodium ion $\left(\mathrm{Na}^{+}\right)$after one week before and after prevention, was statistically significant $(P<0.01)$. And the comparison of results after 3 months prevention and neonatal birth plus one week, was also statistically significant $(P<0.05)$, while differences in the newborn after 3 months prevention, were also statistically significant $(P<0.05)$. In the comparison of the results before and after 3 months of PMTCT, a significant difference was found in Chloride $(P<0.05)$, as was the comparison of newborns after one week before and after prevention $(P<0.01)$. In the comparison of results after 3 months prevention and neonatal birth after one week, the difference was statistically significant $(P<0.05)$. Furthermore, the gap of Anion (BG), before and after 3 months of PMTCT, was found significantly different $(P<0.05)$, as was the comparison of newborns after one week before and after prevention $(P<0.01)$. For serum osmotic pressure (OS), in results before and after 3 months of PMTCT, the difference was statistically significant $(P<0.01)$. For the comparison of newborns after one week before and after prevention, the difference was statistically significant $(P<0.01)$. There was statistically significant between the results after 3 months prevention and neonatal birth plus one week $(t=2.3121, P=0.0107)$. As for serum OS, the comparison of the results before and after 3 months of PMTCT, was statistically significant $(P<0.05)$. Blood urea nitrogen (BUN), in comparison between newborns after one week before and after prevention, the difference was statistically significant $(P<0.05)$. For uric acid (UA), the comparison of results before and after 3 months of PMTCT, was significant $(P<0.05)$, as was the comparison of newborns after one week before and after prevention $(P<0.01)$. There were in Bunlcr, significant differences before and after 3 months of PMTCT $(P<0.01)$, while the comparison between newborns after one week before and after prevention was statistically significant $(P<0.01)$. On the other hand, after detecting the same samples in three groups by repeated analysis of variance, the $P$ values for BUN, creatinine $(\mathrm{Cr})$ and bicarbonate $\left(\mathrm{HCO}_{3}{ }^{-}\right)$were more than 0.05(Table 2). 
Table 2 Comparison of detection results of electrolytes and renal function indexes before and after PMCTC in HIV/AIDS

\begin{tabular}{lccccc}
\hline Group & $\mathrm{K}^{+}(\mathrm{mmol} / \mathrm{L})$ & $\mathrm{Na}^{+}(\mathrm{mmol} / \mathrm{L})$ & $\mathrm{Cl}^{-}(\mathrm{mmol} / \mathrm{L})$ & $\mathrm{BG}(\mathrm{mmol} / \mathrm{L})$ & $\mathrm{OS}(\mathrm{mmol} / \mathrm{L})$ \\
\hline Before PMCTC & $3.96 \pm 0.4$ & $137.93 \pm 3.42$ & $100.77 \pm 3.72$ & $15.26 \pm 3.03$ & $284.08 \pm 6.93$ \\
3 months after PMCTC & $3.95 \pm 0.36$ & $139.7 \pm 2.67$ & $101.57 \pm 3.08$ & $16.02 \pm 3.77$ & $287.6 \pm 5.44$ \\
Newborns after one week & $4.07 \pm 0.39$ & $140.34 \pm 3.03$ & $102.29 \pm 3.25$ & $16.6 \pm 3.51$ & $289.13 \pm 6.08$ \\
$F$ value of CV & 4.58 & 25.37 & 7.78 & 5.77 & 26.69 \\
$P$ value & 0.011 & $<0.001$ & $<0.001$ & 0.003 & $<0.001$ \\
\hline Group & $\mathrm{BUN}(\mathrm{mmol} / \mathrm{L})$ & $\mathrm{Cr}(\mu \mathrm{mol} / \mathrm{L})$ & $\mathrm{UA}(\mu \mathrm{mol} / \mathrm{L})$ & $\mathrm{BUN} / \mathrm{Cr}$ & $\mathrm{HCO}_{3}^{-}(\mathrm{mmol} / \mathrm{L})$ \\
\hline Before PMCTC & $4.63 \pm 1.77$ & $76.18 \pm 51.11$ & $386.23 \pm 160.76$ & $0.06 \pm 0.02$ & $25.85 \pm 2.63$ \\
3 months after PMCTC & $4.78 \pm 1.37$ & $75.96 \pm 32.12$ & $352.77 \pm 129.08$ & $0.07 \pm 0.02$ & $26.06 \pm 2.85$ \\
Newborns after one week & $5.11 \pm 2.16$ & $79.53 \pm 48.12$ & $343.68 \pm 120.69$ & $0.07 \pm 0.02$ & $25.52 \pm 2.86$ \\
$F$ value of CV & 2.84 & 0.31 & 4.01 & 12.67 & 1.46 \\
$P$ value & 0.059 & 0.736 & 0.019 & $<0.001$ & 0.234 \\
\hline
\end{tabular}

\section{Comparison of indicators of liver function before and after PMTCT of HIVIAIDS transmission}

For the comparison of the results of alanine amino transferase (ALT), aspartic acid amino transferase (AST), AST/ALT, alkaline phosphatase (ALP) and indirect bilirubin (IDIL), before and after 3 months of PMTCT, there was no significant statistical difference, while in the comparison of gamma glutamyltransferase (gamma-GT) from newborns after one week before and after prevention, a statistically significant difference was found $(P<0.05)$.

What's more, the comparison of the results of total bilirubin (TDIL) after 3 months prevention and one week post birth, was statistically significant $(P<0.05)$. In the comparison of direct bilirubin (DBIL) before and after 3 months of PMTCT, the result was, statistically significant $P<0.01$, while the comparison of results before and after one week of newborn prevention was, also statistically significant $P<0.05$.

As for total protein (TP), the comparison of results after 3 months prevention was statistically significant $(P<0.05)$, and the comparison of newborns after one week before and after prevention, was also statistically significant $(P<0.05)$. As for albumin (ALB), results before and after 3 months of PMTCT showed significant differences $(P<0.01)$, while the comparison of newborns after one week before and after prevention also showed $P<0.01$. In the comparison of results after 3 months prevention and neonatal birth of one week, $P<0.05$ was found. After detecting the same samples in three groups by repeated analysis of variance: except for direct bilirubin (DBIL) and albumin (ALB), there was no significan in other indicators. (Table 3).

\section{Comparison of main biochemical indexes before and after PMTCT of HIVIAIDS to newborns in pregnant women}

For random blood glucose (Glu), triglyceride (TG), total cholesterol (TC), amylase (AMY) and lactic acid (LACT) before and after 3 months of PMTCT, there was no significant difference. However for creatine kinase (CK) before and after 3 months of PMTCT, there were significant differences $(P<0.05)$. For newborns after one week before and after prevention, the difference was also statistically significant $(P<0.01)$ The comparison of results after 3 months prevention with one week after birth was statistically significant $(P<0.05)$. When it came to lactate dehydrogenase (LDH) before

Table 3 Comparison of PMTCT indicators of liver function of HIV/AIDS pregnant $(\bar{x} \pm s)$

\begin{tabular}{|c|c|c|c|c|c|}
\hline Group & ALT(U/L) & $\mathrm{AST}(\mathrm{U} / \mathrm{L})$ & AST/ALT & ALP(U/L) & $\gamma-\mathrm{GT}(\mathrm{U} / \mathrm{L})$ \\
\hline Before PMCTC & $27.9 \pm 24.46$ & $30.86 \pm 18.27$ & $1.30 \pm 0.50$ & $100.61 \pm 53.86$ & $114.81 \pm 134.35$ \\
\hline 3 months after PMCTC & $25.36 \pm 21.8$ & $28.37 \pm 20.81$ & $1.29 \pm 0.50$ & $100.90 \pm 45.30$ & $93.45 \pm 132.45$ \\
\hline Newborns after one week & $25.77 \pm 21.41$ & $27.97 \pm 20.89$ & $1.24 \pm 0.47$ & $99.89 \pm 33.23$ & $84.07 \pm 103.68$ \\
\hline$F$ value of $\mathrm{CV}$ & 0.55 & 0.93 & 0.65 & 0.02 & 2.44 \\
\hline$P$ value & 0.575 & 0.396 & 0.521 & 0.980 & 0.088 \\
\hline Before PMCTC & $8.87 \pm 7.34$ & $4.16 \pm 4.18$ & $4.71 \pm 3.91$ & $77.32 \pm 7.42$ & $42.68 \pm 6.89$ \\
\hline 3 months after PMCTC & $7.49 \pm 4.14$ & $3.27 \pm 1.60$ & $4.21 \pm 2.98$ & $78.81 \pm 6.5$ & $45.05 \pm 5.15$ \\
\hline Newborns after one week & $7.82 \pm 4.46$ & $3.33 \pm 2.40$ & $4.49 \pm 2.74$ & $78.82 \pm 6.88$ & $46.15 \pm 5.78$ \\
\hline$F$ value of $\mathrm{CV}$ & 2.60 & 4.37 & 0.90 & 2.35 & 13.35 \\
\hline$P$ value & 0.075 & 0.013 & 0.406 & 0.097 & $<0.001$ \\
\hline
\end{tabular}


and after 3 months of PMTCT, the $P$ value was less than 0.01 , showing significant difference, while the comparison of newborns after one week before and after prevention, was statistically significant $(P<0.01)$. In high density lipoprotein cholesterol ester (HDL) before and after 3 months of PMTCT, there were significant differences $(P<0.01)$, while the comparison of newborns after one week before and after prevention, was statistically significant $(P<0.01)$. For the comparison of results after 3 months prevention with neonatal birth of one week, the differences were statistically significant $(P<0.01)$. In low density lipoprotein cholesterol (LDL) before and after 3 months of PMTCT, there were significant differences $(P<0.01)$. In the comparison of newborns after one week before and after prevention, the difference was also statistically significant $(P<0.01)$. Comparision of indexes between 3 months after interruption and one week after birth were statistically significant $(P<0.05)$. After detecting the same samples in three groups by repeated analysis of variance, there were significant differences in $\mathrm{CK}$, LDH, HDL and LDL $(P<0.05$, Table 4$)$.

Table 4 Comparison of other main biochemical indexes before and after PMCTC in HIV/AIDS $(\bar{x} \pm s)$

\begin{tabular}{lccccc}
\hline Group & GLU(mmol/L) & CK(U/L) & LDH(U/L) & TG(mmol/L) & TC(mmol/L) \\
\hline Before PMCTC & $5.61 \pm 1.43$ & $89.92 \pm 75.05$ & $329.74 \pm 76.69$ & $1.96 \pm 2.09$ & $4.74 \pm 1.31$ \\
3 months after PMCTC & $5.54 \pm 1.22$ & $105.15 \pm 67.27$ & $216.56 \pm 58.56$ & $2.11 \pm 2.43$ & $4.70 \pm 1.22$ \\
Newborns after one week & $5.56 \pm 1.33$ & $119.03 \pm 69.57$ & $219.25 \pm 62.51$ & $2.03 \pm 2.00$ & $4.69 \pm 1.20$ \\
$F$ value of CV & 0.11 & 6.45 & 143.89 & 0.18 & 0.07 \\
$P$ value & 0.894 & 0.002 & $<0.001$ & 0.835 & 0.934 \\
\hline Group & HDL $(\mathrm{mmol} / \mathrm{L})$ & $\mathrm{LDL}(\mathrm{mmol} / \mathrm{L})$ & AMY(U/L) & LACT(mmol/L) \\
\hline Before PMCTC & $1.36 \pm 0.68$ & $2.99 \pm 1.05$ & $92.38 \pm 37.00$ & $4.26 \pm 2.41$ \\
3 months after PMCTC & $1.92 \pm 0.92$ & $2.36 \pm 1.19$ & $95.22 \pm 40.48$ & $4.23 \pm 2.92$ \\
Newborns after one week & $2.18 \pm 1.01$ & $2.11 \pm 1.11$ & $89.18 \pm 32.81$ & $3.93 \pm 2.91$ \\
$F$ value of CV & 34.38 & 25.00 & 1.02 & 0.67 \\
$P$ value & $<0.001$ & $<0.001$ & 0.362 & 0.514
\end{tabular}

\section{DISCUSSION}

There are three critical periods of time in PMTCT, namely prevention: intrauterine, intrapartum and postpartum. Antiviral treatment is one of the best methods of HIV PMTCT implementation, and is the preferred solution for mother to child disease transmission prevention. Once this approach is settled upon, an appropriate mode of delivery is required. Artificial feeding, instead of breast feeding, is the last line of defense for mother to child disease prevention ${ }^{[5,12-13]}$. The transmission of HIV / AIDS from mother to child is one of the three ways by which AIDS can be transmitted. If a pregnant woman with HIV cannot be treated in time, it is highly likely that the newborn will be infected. Therefore, the application of PMTCT is essential. If HIV is found early during pregnancy, consultation with an appropriate specialist is necessary, which will give the mother information and / or treatment to either continue the pregnancy or terminate. During the continued pregnancy, antiviral treatment and appropriate methods of delivery in hospital are highly recommended. At the same time, artificial feeding has to take place of breastfeeding after birth, in order to administer PMTCT. After 12 months, HIV antibody is tested, in order to determine whether the PMTCT has been successful. A large amount of research shows that if AIDS pregnant women fail to carry out immediate PMTCT, the MTCT (mother to infant HIV / AIDS) transfer rate can reach from $30 \%$ to $40 \%$. On the contrary, if PMTCT is carried out timely and successfully throughout the required period, the mother to child transmission rate can be reduced to $2 \%$ to $3 \%^{[14-17]}$. In this study, after carrying out PMTCT in 152 cases of HIV / AIDS in pregnant women, neonatal infection was eliminated.

Although PMTCT can prevent mother to child transmission, unfortunately AIDS antiviral / antiretroviral drug treatment has varying side effects. This may be adverse to the normal development of the fetus, or in some cases cause malformation. As for the woman, these may be poor blood circulation, liver and renal function damage and gastrointestinal reaction ${ }^{[10-11]}$. Therefore, much attention should be attached to the careful use of PMTCT.

There was no significant difference in viral load before and after PMTCT in three groups $(P>0.05)$, indicating that there were nearly no changes in virus load in participants overall, as most of the patients remained stable. Virus load changed greatly in a few patients, from large values to normal, and viral load increased continuously up to tens of thousands of times after prevention in patients of a small number. 
As for T lymphocyte count, the indexes of CD4 and CD4/CD8 in newborns before and after one week prevention, were statistically different $(P<0.05)$, while for other groups there were no statistically significant differences. The results show that after PMTCT, the patient's immune systems can be greatly strengthened, with CD4 increased significantly, which indicates that the mother and infanthave different responses to PMTCT.

The electrolytes and renal function of pregnant women with HIV/AIDS before and after PMTCT, $\mathrm{K}^{+}$ in the groups of newborns before and after PMTCT prevention, and three months post PMTCT prevention compared with the newborns after one week, were statistically significant $(P<0.01)$, which can explain why $\mathrm{K}^{+}$remains at a low level before and after 3 months prevention, while it gradually increased to normal and relative stable levels after birth. In the comparison of $\mathrm{Na}, \mathrm{Cl}^{-}$, OS before and after three months prevention, and newborns after a week, there were significant differences $(P<0.05)$. It indicates that $\mathrm{Na}^{+}, \mathrm{Cl}^{-}$, OS before prevention was at a lower level, because the invasion of HIV causes the disorder both inside and outside ionic and osmotic balance. After three months of PMTCT, $\mathrm{Na}^{+}, \mathrm{Cl}^{-}$, OS can reach the certain level, while with the continuation of PMTCT in newborns at one week, $\mathrm{Na}^{+}, \mathrm{Cl}$, OS increased to normal levels, maintained at a certain level. Comparing BG, UA, BUN/Cr before and after 3 months PMTCT prevention with newborns at one week before and after 3 months PMTCT prevention, the differences were statistically significant $(P<0.05)$, while the result after 3 months and after one week in newborns, was not statistically signifi cant $(P>0.05)$. The tests showed that BG, UA, BUN/Cr before PMTCT prevention remains at the same(high or low) level. However, after three months prevention and newborns after one week, it increased to a high level. Therefore, there was no significant difference in the test results at three months and newborns after one week. Comparing the results of BUN before and after PMTCT prevention, there were statistical differences $(P<0.05)$. With the deep prevention, the concentration increased slowly, while after one week in newborns, it was stable. Other indicators, such as $\mathrm{Cr}$ and $\mathrm{HCO}_{3}{ }^{-}$had no statistical significance before and after treatment $(P>0.05)$. In summary, the results showed that it is much more prudent to study PMTCT of HIV/AIDS pregnancies due to its negative influence on the balance of electrolytes and renal function.

The results of this study confirmed that in the test result of liver function of HIV/AIDS pregnant mother and child, there were significant differences in ALB before and after three months prevention, and newborns after one week prevention $(P<0.05)$, which indicates that during the invasion from the HIV virus, ALB is over-consumed before PMTCT, leading it to a relatively low level, while with the depth of PMTCT before and after prevention, ALB gradually increased and reached a higher level. From this perspective, PMTCT demonstrates a positive relationship with the rise of CD4. DBIL and TP before and after three months prevention were compared with newborns before and after one week prevention, showing statistically significant differences $(P<0.05$ or above $)$. However, when the results after three months prevention was compared with newborns after one week prevention, there was no statistical difference $(P>0.05)$. This result explains why DBIL and TP before PMTCT prevention remains at a high or low level. However, after three months prevention and newborns after one week, it reached a high or low level and kept to a stable level. Therefore, there was no significant difference in the results between three months after PMTCT and newborns after one week prevention. there were statistical differences in TDIL before and after PMTCT prevention $(P<0.05)$. This showed that due to the HIV virus, TDIL kept a relatively high level before PMTCT, and with the in-depth prevention, the concentration reduced to a normal level and kept at this level. Among the selected patients, except indicators mentioned above had significant differences, other indexes of liver function before and after prevention were not statistically significant $(P>0.05)$. Repeated detection error analysis was carried out for the same subjects in three groups. The results showed that PMTCT in HIV/AIDS had a slight influence on mothers' liver function.

In the test results of other main biochemical indexes, CK, HDL and LDL etc., before and after three months of PMTCT and newborns of one week after PMTCT, statistical significance was found $(P<0.05)$. This can be explained by the invasion of HIV causing $\mathrm{CK}, \mathrm{HDL}$ and LDL remaining at a low or high level prior to prevention. After three months of PMTCT, $\mathrm{CK}, \mathrm{HDL}, \mathrm{LDL}$ can increase or reduce to the certain level, while with the deepening of PMTCT to newborns of one week, CK, HDL, LDL can be increased or reduced to normal levels. Comparing LDH before and after 3 months of PMTCT prevention with newborns of one week before and after 3 months PMTCT prevention, the differences were statistically significant $(P<0.01)$, while there was no significant difference in results of after 3 months PMTCT in mothers and after one week in newborns $(P>0.05)$. The test result showed that LDH before PMTCT prevention remains at a high or low level. However, after three 
months prevention, it increased to a high or stable level. Therefore, there was no significant difference in mothers after three months PMTCT and newborns after one week prevention. Comparing other major biochemical indexes, such as GLU, TG, TC, AMY and LACT before and after PMTCT prevention, there was no statistical difference $(P<0.05)$, which indicates that PMTCT has little influence on major biochemical indexes and is worthy of study in order to provide good basis for clinical medicine practice.

Patients enrolled in this study were treated in the local hospital. Therefore, the results can only reflect the local area and do not necessarily represent the situation throughout China as a whole, leading to inherent quality control limitations.

The dynamic analysis of viral load, T lymphocyte subsets and main biochemical indexes before and after PMTCT in HIV/AIDS will be an important method to evaluate the dose and treatment of antiretroviral drugs. Monitoring those indexes can help to judge and analyze the condition of the maternal body at various stages, and then adjust the antiviral drugs. If there are changes in biochemical indicators, the dose of antiviral drugs will need to be optimized. If the indicator remains at the original level, antiviral drugs need to remain at the original dose in order to achieve the best therapeutic scheme, as well as improving the production rate and patients' quality of life.

\section{Acknowledgments and Funding}

During the process of this research, we got much help from many departments and individuals, such as the city health bureau leadership and technology bureau leadership, and the participating hospital's Infectious Diseases and Infection Clinic, as well as other organizations not directly involved in the research. All of them offered a great support and help in this research, and the research group showed deepest appreciation to them.

The study was supported by the fund of the Guangxi Zhuang Autonomous Region Health Committee (No. Z2014574) and the development project of Nanning Science and Technology Bureau (No.20143154).

\section{References}

[1] Haile ZT, Teweldeberhan AK, Chertok IR. Correlates of women's knowledge of mother-to-child transmission of HIV and its prevention in Tanzania:a population-based study[J]. AIDS Care, 2016,28(1):70-78

[2] Balogun FM, Owoaje ET. Perception about the'Opt Out Strategy'for HIV testing and counselling among pregnant women attending antenatal clinic in Ibadan,Nigeria[J]. Journal of Community Medicine and Primary Health
Care, 2016,28(1):45-51

[3] Ngoma-Hazemba A, Ncama BP. Analysis of experiences with exclusive breastfeeding among HIV-positive mothers in Lusaka, Zambia[J]. Glob Health Action,2016,9(1):1-8

[4] Stringer EM, Ekouevi DK, Coetzee D, et al. Coverage of nevirapine-based services to prevent motherto-child HIV transmission in 4 African countries[J]. JAMA,2010,304(3):293-302.

[5] Dionne-Odom J, Massaro C, Jogerst KM, et al. Retention in care among HIV-infected pregnant women in haiti with PMTCT option B[J]. AIDS Res Treat,2016,2016:6284290.

[6] Psaros JE, Smit JA. Adherence to HIV care after pregnancy among women in sub-saharan Africa:falling off the cliff of the treatment cascade[J]. Curr HIV/AIDS Rep,2015,12(1):1-5

[7] Ambia J, Mandala J. A systematic review of interventions to improve prevention of mother-to-child HIV transmission service delivery and promote retention[J]. J Inter AIDS Soc, 2016,19(1) : 20309

[8] Chi BH, Tih PM, Zanolini A, et al. Implementation and operational research:reconstructing the PMTCT cascade using cross-sectional household survey data:the PEARL study[J]. J Acquir Immune Defic Syndr, 2015,70(1):e5-e9

[9] J Wadams KA, Momplaisir FM. Postpartum engagement in HIV care:an important predictor of long-term retention in care and viral suppression[J]. Clinical Infectious Diseases, 2015,61(12):1880-1887

[10] Gao J, Wu YL, Zou Y, et al. Analysis of comprehensive intervention measures for pregnant women with HIV infection from 2012 to 2016 in Hunan province[J]. Chinese Journal of Maternal and Child Health, (in Chinese), 2017,8(5):14-18

[11] Luo MH, Tang XJ, Zhang J, et al. Evaluation of the effect of mother to child AIDS prevention program in Laibin city, Guangxi Zhuang Autonomous Region [J]. China Tropical Medicine, 2017,17 (6): 601-603+615(in Chinese).

[12] Kashitala J, Nyambe N, Mwalo S,et al. Is male involvement in ANC and PMTCT associated with increased facility-based obstetric delivery in pregnant women? [J]. Afr J Reprod Health, 2015 , 19(2):117-24.

[13] Mandelbrot L, Tubiana R, Le Chenadec J, et al. No perinatal HIV-1 transmission from women with effective antiretroviral therapy starting before conception [J]. Clinical Infectious Diseases, 2015, 61(11): 1715-1725.

[14] Pang J, Liu DM, Ge L, et al. Evaluation of the effect of antiretroviral therapy on mother to child transmission of HIV and AIDS during pregnancy and intrapartum[J]. Chinese AIDS and sexually transmitted diseases, 2017, 23(8):723725

[15] Peng LQ. Correlation between antiretroviral therapy and mother to child transmission of human immunodeficiency virus in pregnant women with HIV/AIDS[J]. Chinese Medical Sciences, 2017,7(3):179-181 
A dynamic analysis of viral load,T lymphocyte subsets and main biochemical indexes before and after prevention of mother to child transmission in HIV/AIDS, 2018, 2(4)

[16] Ndege S, Washington S, Kaaria A, et al. HIV prevalence and antenatal care attendance among pregnant women in a large home-based HIV counseling and testing program in Western Kenya[J]. PLoS One, 2016,11(1):e0144618

[17] President's Emergency Plan for AIDS Relief. PEPFAR
Country/Regional Operational Plan (COP/ROP) 2016 Guidance, 2016[EB/OL]. https://www.popline.org/ node/637035

(Received 04 September 2018, Revised 08 October 2018, Accepted 04 November 2018) 


\section{Antibodly}

安迪浭㨋

\section{JiangSu LIBO Medicine Biotechnology Co.,Ltd.}

\section{Diagnosis of Hemolytic Disease of the Newborn}

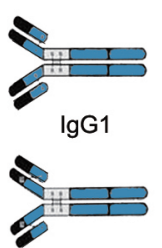

$\lg \mathrm{Ig}$

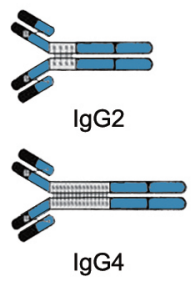

\section{IgG Subclasses Test Card}

The product is used for prediction of Hemolytic Disease of the Newborn, including the detection of Anti-A(B) IgG Subclasses of the Newborn Red Blood Cell and Pregnant Women Serum.

\section{Treatments for Hemolytic Diseases of the Newborn(Fetus) (For Reference Only)}

Pre-pregnancy — Effective Intervention

- Blood typing

- Detection of antibody potency

- Combination of traditional chinese and westem medicine treatment

- Plasmapheresis (Removal)

- High dose of gammaglobulin

\section{Pregnancy — Active Treatment}

- Monitor of antibody potency

- Combination of traditional chinese and westem medicine treatment

- Plasmapheresis (Removal)

- High dose of gammaglobulin

- Intrauterine transfusion

\section{Postpartum — Symptomatic Treatment}

- Hemolysis detection

- Phenobarbital

- Phototherapy

- Gammaglobulin and hormone

- Albumin

- Exchange transfusion in neonates
Early Treatment for Pregnant Women with High Potency

Traditional Chinese Medicine

- Herba Artemisiae Scopariae, Radix Astragali, Fructus Gardeniae

- Herba Taxilli, Semen Cuscutae, Radix Dipsaci Asperoidis

- Radix Angelicae Sinensis, Radix Paeoniae ALBA

- Rhizoma Atractylodis Macrocephalae

\section{Western Medicine}

- Vc: 100mg,1/d;

- VE: $100 \mathrm{mg}, 1 / \mathrm{d}$;

- Oxygen inhalation: 20min per time, 2/d; Plasma(Lymph) Exchange, Immunosorbent Intrauterine Transfusion

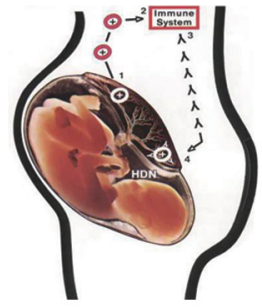

\section{Antibod 进迪溥据 \\ JiangSu LIBO Medicine Biotechnology Co.,Ltd.}

Address: No 78 West Dongsheng Road, Jiangyin, Jiangsu214400, P.R. China

Web Site: www.libiotech.com

Sales Tel: +86-510-86990618、+86-510-86990633

Tech Tel: +86-510-86990608、+86-510-86990655

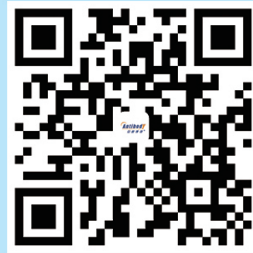

